\title{
Target-Focused Feature Selection Using Uncertainty Measurements in Healthcare Data
}

\author{
ORPAZ GOLDSTEIN, MOHAMMAD KACHUEE, KIMMO KARKKAINEN, and \\ MAJID SARRAFZADEH, University of California, Los Angeles
}

\begin{abstract}
Healthcare big data remains under-utilized due to various incompatibility issues between the domains of data analytics and healthcare. The lack of generalizable iterative feature acquisition methods under budget and machine learning models that allow reasoning with a model's uncertainty are two examples. Meanwhile, a boost to the available data is currently under way with the rapid growth in the Internet of Things applications and personalized healthcare. For the healthcare domain to be able to adopt models that take advantage of this big data, machine learning models should be coupled with more informative, germane feature acquisition methods, consequently adding robustness to the model's results. We introduce an approach to feature selection that is based on Bayesian learning, allowing us to report the level of uncertainty in the model, combined with false-positive and false-negative rates. In addition, measuring target-specific uncertainty lifts the restriction on feature selection being target agnostic, allowing for feature acquisition based on a target of focus. We show that acquiring features for a specific target is at least as good as deep learning feature selection methods and common linear feature selection approaches for small non-sparse datasets, and surpasses these when faced with real-world data that is larger in scale and sparseness.
\end{abstract}

CCS Concepts: • Computing methodologies $\rightarrow$ Machine learning; Feature selection; $\bullet$ Applied computing $\rightarrow$ Health informatics; • Mathematics of computing $\rightarrow$ Bayesian networks;

Additional Key Words and Phrases: Healthcare feature selection, healthcare big data, machine learning, machine learning for health, health informatics, Bayesian learning

ACM Reference format:

Orpaz Goldstein, Mohammad Kachuee, Kimmo Karkkainen, and Majid Sarrafzadeh. 2020. Target-Focused Feature Selection Using Uncertainty Measurements in Healthcare Data. ACM Trans. Comput. Healthcare 1, 3, Article 15 (May 2020$), 17$ pages. https://doi.org/10.1145/3383685

\section{INTRODUCTION}

As big data becomes ubiquitous, so does the increase in the dimensionality of data. As the selection of features increases, feature selection becomes a necessary tool in the evaluation and acquisition of features [26], and in turn for the training of learning models. This is increasingly true in the healthcare domain, where data is accumulated and under-utilized [13,35,40]. Moreover, in the healthcare domain, both budget for features and model uncertainty should be taken into account for a feature selection model to be practical. Since in many cases our main target of interest is the minority target, we would rather focus on reducing the uncertainty of a specific

Authors' address: O. Goldstein, M. Kachuee, K. Karkkainen, and M. Sarrafzadeh, University of California, Los Angeles; emails: \{orpgol, mkachuee, kimmo, majid\}@cs.ucla.edu.

Permission to make digital or hard copies of all or part of this work for personal or classroom use is granted without fee provided that copies are not made or distributed for profit or commercial advantage and that copies bear this notice and the full citation on the first page. Copyrights for components of this work owned by others than ACM must be honored. Abstracting with credit is permitted. To copy otherwise, or republish, to post on servers or to redistribute to lists, requires prior specific permission and/or a fee. Request permissions from permissions@acm.org.

(C) 2020 Association for Computing Machinery.

$2637-8051 / 2020 / 05-A R T 15 \$ 15.00$

https://doi.org/10.1145/3383685

ACM Transactions on Computing for Healthcare, Vol. 1, No. 3, Article 15. Publication date: May 2020. 
target of interest rather than the general uncertainty while maintaining a budget for features. In this article, we propose an approach for feature selection that is based on improving model confidence on a single target out of many, allowing us exceptional frugality in feature acquisition.

Incorporating machine learning models in extreme accuracy inclined environments such as the healthcare domain requires some adaptation in the way information is acquired and incorporated into a model, as well as how the model informs the healthcare provider of its observations [40, 41]. Real-world constraints on data collection and availability, as well as non-uniform importance distribution on target features of a model, should be taken into account when constructing a model under such real-world conditions [29]. When dealing with data that is not all available up front, our assumptions toward it might change as new data comes in. New features might appear to carry more weight or contradict our previous assumptions of feature importance. In addition, although model precision is normally used as the main metric we strive to improve when selecting features for our machine learning models, some situations call for more attention to minimizing model uncertainty. In a scenario where data is coming in iteratively as it is being acquired, it makes sense to measure uncertainty to understand when is an acceptable time to make predictions and when we should ask for more data. Users who need highly accurate models can be more confident with the models when they know the uncertainty rates, and it allows them to reason whether the model is likely to make accurate predictions consistently. Since in many cases our main target of interest is the minority target, we focus on reducing the uncertainty of a specific target of interest class in our model, allowing us to be extra careful with our budget. For example, two types of heart disease might display similar symptoms, but we rather focus our resources on understanding whether the patient has a less common disease that is more fatal. Normally, features will be evaluated based on their contribution toward both heart diseases. In our approach, we acquire features that are more informative toward the prediction of a single target.

Feature selection is the process of acquiring a subset of features that are considered most informative to a machine learning model out of all available features. In many real-world situations, features have some associated cost to them and therefore should be selected based on both how informative they are and the cost they incur. Features are normally selected based on some global metric of information gain. When the need for accuracy is extreme and the cost of features is high, it might make sense to limit the feature information gain measurement to a per-class investigation-that is, limiting our feature selection process to acquiring features that assist most in the prediction/classification of one specific target out of many. In this way, we can be extremely frugal in paying for features while making sure we are contributing to the classification of a higher importance target.

Uncertainty measurement in a machine learning model flows from applying a probabilistic approach to learning, also known as Bayesian learning [32]. Sampling a trained probabilistic model for latent variables allows us to capture the inherent uncertainty in the model. Identifying the uncertainty within a model is important for being able to give accurate guarantees for what a model can efficiently predict [24, 25, 37]. For example, trying to predict between two types of heart disease, one type could be more common and make most of the samples available to us. Drilling into the results could show that the less common but more fatal disease is showing high variance in the classification predicted by the model for that class, indicating uncertainty in the more fatal type of heart disease. Developing models that are minimally uncertain of their predictions is the key to incorporating machine learning in domains such as healthcare. The usage of Gaussian weight distributions to estimate the uncertainty was first discussed in Denker and LeCun [6]. Later work includes Bishop [3], Ghahramani [11], and Murphy [30], and many more. The benefits of uncertainty measurement in the healthcare domain flow from the statistical rigor of the outputs provided by a model. In Krzywinski and Altman [24], the importance of minimizing uncertainty in biology is discussed, and the work in Herzog and Ostwald [15] calls biologists to shift to Bayesian statistics and provides reasoning for the need for uncertainty measurements in this field.

In this article, we propose a novel probabilistic uncertainty-based method for target-specific feature acquisition. The main contributions of this work are as follows: 
- Our first contribution is providing a method to focus resources on a single target of interest that is generalizable, scalable, and consistent in selecting informative features for a specific single target of interest out of many.

- Our second contribution is a polynomial-time, threshold-based method, allowing us to reason on model confidence in predictions while learning a representation of the data, and to make a decision on whether to ask for more data or declare readiness to start making predictions on real-world scenarios.

\section{RELATED WORK}

Classic approaches to feature selection focus on maximizing information gain and inferring feature relevance $[2,14]$. Health informatics methods of feature selection take into account real-world costs associated with the acquisition of features and the need to maintain a budget. Costs of tests, physician time, and patient discomfort should all be taken into account when reasoning on which feature is to be acquired, by using cost-sensitive decision methods or active sensing $[8,42]$. In addition to costs, changes in medical data availability might call for iterative feature aggregation in training time, requiring an online cost-sensitive budgeted approach $[9,12$, 20]. When managing a budget for features acquired iteratively, a deep reinforcement learning (DRL) approach for optimizing a trade-off between the expected classification error and the feature cost was recently suggested [17]. Although it performs well for feature acquisition tasks, reinforcement learning usually runs for long periods before outputting a decision.

Healthcare data tends to be imbalanced, and some conditions or variants of a disease are more common. Some targets carry more significance or are more relevant to a specific diagnosis. The acquisition of relevant data is made possible using an active learning approach [31, 39], or by reducing redundancy in acquired features while maintaining relevance $[18,28]$. Contributing to the imbalance is also the sparseness of data. Due to the high dimensionality of the data, not all data points will have all features. For medical domain feature selection and prediction, ensemble methods have been used to reduce the effects of imbalanced data, and of inherent missingness $[16,27]$, and more recently with a robust feature selection framework [43]. Although addressing the imbalance in data is closely related to our work, the acquisition of features that are germane to a specific target of focus is not addressed. The acquisition of a massive amount of features in the medical domain was explored in [44].

Application of uncertainty to feature selection robustness appears in same-decision probability (SDP) [4], which measures the effect of feature acquisition on the shift in the decision making of a model. SDP measures the uncertainty in the model while acquiring features and reasons on stopping criteria based on a threshold of confidence and budget. More recently, an expected SDP query and an optimal feature selection algorithm based on SDP were proposed [5]. SDP queries are generally $P P^{P P}$-complete, which makes it costly for many high-dimensional real-world applications.

\section{NOTATION}

The following notation is used throughout the article. $\mathbb{X}$ represents all data available to us at a given moment, $\mathrm{X}$ represents an instantiated subset of all available observations data, made up of data points of size $r$ and feature set $\mathbf{F}=\left\{F_{1} \ldots F_{c}\right\}$ of size $c$ such that $\mathbf{X} \in \mathbb{R}^{r, c} . \lambda$ represents the free parameters $\mathbf{W}$ and $\mathbf{b}$ (weight and bias) that are learned by the model trying to estimate the latent variables. The true posterior distribution for our latent variables is represented by $\mathbf{z}$ (i.e., the distribution of true targets that is unknown to us). The estimated posterior is represented by y. $\theta$ represents a specific target class out of all potential targets. $\mathbf{t}_{\theta}$ denotes the true target vector for a target class $\theta$ and $\mathbf{y}_{\theta}$ a subset of our predictions corresponding to the true targets in $\mathbf{t}_{\theta}$.

\section{BACKGROUND}

To capture uncertainty in a model, we need to learn a representation of a latent distribution over a set of parameters defining that distribution and be able to sample the learned parameters to associate the captured uncertainty 
with test time examples of the data and targets. Our optimization function therefore will be taking a probabilistic approach.

Using variational inference, we will estimate $\lambda^{\star}$ using Kullback-Leibler (KL) divergence such that

$$
\lambda^{\star}=\operatorname{argmin}_{\lambda} K L(q(\mathbf{z} ; \lambda) \| p(\mathbf{z} \mid \mathbf{X})),
$$

where $q(\mathbf{z} ; \lambda)$ is the estimation of posterior distribution $p(\mathbf{z} \mid \mathbf{X})$ optimized over parameters $\lambda$.

Since the posterior $p(z \mid X)$ is unknown to us, we will resort to maximizing the evidence lower bound (ELBO) as an optimization function:

$$
\operatorname{ELBO}(\lambda)=\mathrm{E}_{q(\mathbf{z} ; \lambda)}[\log p(\mathbf{X}, \mathbf{z})-\log q(\mathbf{z} ; \lambda)],
$$

which is equivalent to minimizing KL divergence [3, 19, 22].

Gradient optimization of ELBO is done via the reparameterization trick [22]:

$$
\nabla_{\lambda} E L B O(\lambda) \approx \frac{1}{s} \sum_{s=1}^{s}\left[\nabla_{\lambda}(\log p(\mathbf{X}, \mathbf{z}(\epsilon ; \lambda))-\log q(\mathbf{z}(\epsilon ; \lambda) ; \lambda))\right],
$$

where $s$ is the number of samples drawn.

\section{TARGET-FOCUSED FEATURE SELECTION}

\subsection{Problem Setup}

Using a minimal amount of features, our goal is to achieve reasonable confidence for a specific class, as described in our objective function:

$$
\operatorname{argmax}_{F S}\left(\text { confidence }_{\theta}-\sum_{f_{i} \in F S} \frac{1}{v_{i}}\right) \text {, }
$$

Subject to : $|F S|<\beta$.

Such that $F S$ is the set of acquired features we wish to minimize, $|F S|$ is the cardinality of the set, and $v_{i}$ is the value associated with each feature. The objective is to frugally acquire the most valuable features while achieving maximum confidence in a specific class $\theta$, without exhausting our budget for features $\beta$.

\subsection{Feature Value Measurement and Acquisitions}

Evaluation of features per target considers the contribution of each feature toward minimizing uncertainty for our target of interest, jointly evaluated with the features already acquired. In addition to confidence scores, features vectors are scored for their cosine similarity, as well as their Hamming weight scores, to gauge potential information gain from a candidate feature.

To use $E L B O$ as our optimization function, we model the linear regression case in which our $\lambda$ contains the input $\mathbf{X}$, a single layer of weights $\mathbf{W}$ and a bias $\mathbf{b}$ such that $\lambda=(\mathbf{W}, \mathbf{b}, \mathbf{X})$. Here, $\mathbf{X} \in \mathbb{R}^{c, r}, w \in \mathbb{R}^{c, d}, b \in \mathbb{R}^{1, d}$. $\mathbf{X}$ has $r$ data points and $c$ features, and the model will learn the distribution over $d$ targets. Assuming independence given our parameters:

$$
p(\mathbf{z} \mid \mathbf{W}, \mathbf{b}, \mathbf{X})=\prod_{n=1}^{r} p\left(\mathbf{z}_{n} \mid \mathbf{X}_{n}^{\top} \mathbf{W}+\mathbf{b}, \sigma_{z}^{2}\right),
$$

where $z$ is the $E L B O$ optimized posterior estimation. We define the priors on both parameters to be the standard normal distribution. 
5.2.1 Measuring Per-Target Uncertainty. Our available data is split into a training set $\mathrm{X}_{\text {train }}$ and a testing set $\mathbf{X}_{\text {test }}$. To obtain our input $\mathbf{X}$, we sample the training data $\mathbf{X}_{\text {train }}$ in a balanced way. For example, if we are trying to predict three targets, then $\mathrm{X}$ will have $33 \%$ of the data points correspond to each of our targets, regardless of the original distribution. To generate a validation input dataset $\mathbf{X}^{\prime}$, we sample $\mathbf{X}_{\text {train }}$ according to its original distribution (no balancing).

At each iteration, a subset of all available features $f_{i} \cup F S$ is trained to learn $\lambda=(\mathbf{W}, \mathbf{b}, \mathbf{X})$. Once trained, we score the feature subset on the validation set $\mathrm{X}^{\prime}$ by measuring the effect acquired features had on a per-target uncertainty. Using our learned distribution, we sample each of our parameters such that $\mathbf{W}^{\prime} \sim \mathbf{W}^{\prime} \mathbf{b}^{\prime} \sim \mathbf{b}$ and calculate the probability vector:

$$
\operatorname{prob}=\operatorname{softmax}\left(\mathbf{X}^{\prime \top} \mathbf{W}^{\prime}+\mathbf{b}^{\prime}\right)
$$

where prob $\in \mathbb{R}^{r, d}$ has the probability of each data point belonging to each possible target. We then get the prediction vector by calculating softmax for each prob $_{i}$ :

$$
\mathbf{y}_{i}=\operatorname{argmax}\left(\operatorname{prob}_{i}\right)=\frac{\exp \left(\operatorname{prob}_{i}\right)}{\sum_{d} \exp \left(\operatorname{prob}_{i, d}\right)} .
$$

Next we evaluate precision, represented by the fraction of times that $\mathbf{y}_{\theta}$ corresponding to target $\theta$ was equal to the correct target for position $i$. Note that $\mathbf{y}_{\theta} \in \mathbf{y}$ and is of subset size $\left|\mathbf{y}_{\theta}\right|$ :

$$
\text { precision }_{\theta}=\frac{1}{\left|\mathrm{y}_{\theta}\right|} \sum_{i=1}^{\left|\mathrm{y}_{\theta}\right|} 1\left(\mathrm{y}_{\theta, i}=\theta\right),
$$

where $1\left(\mathbf{y}_{\theta, i}=\theta\right)$ equals 1 if data point $\mathbf{y}_{\theta, i}$ has the target value $\theta$, and 0 otherwise.

Repeating (6) through (8) for $l$ iterations, sampling the distribution of our parameters each time, our confidence score becomes the averaged precision over multiple iterations. Therefore, the confidence for a specific target is

$$
\text { confidence }_{\theta}=\frac{1}{l} \sum_{j=1}^{l}\left(\text { precision }_{\theta, j}\right) \text {. }
$$

Here, $l$ is the number of times we sample our learned distributions. The trade-off using $l$ is between a more accurate representation of the model confidence and a faster model. We have found that 300 iterations were accurate enough in reporting confidence in our case.

5.2.2 Adding Vector Similarity Scores. In addition to the confidence scores, we wish to capture the potential information gain of the current candidate feature $f_{i}$ given the existing features in FS. We use the computed similarity scores: co-variance distance score and cosine similarity score. We sum the inverse scores for all such pairwise comparisons and then normalize to the range $[0,1]$ :

$$
\begin{aligned}
& \text { CovScore }=N^{0,1} \sum_{g_{i} \in F S} 1-\operatorname{cov}\left(g_{i}, f_{i}\right), \\
& \text { CosScore }=N^{0,1} \sum_{g_{i} \in F S} 1-\cos \left(g_{i}, f_{i}\right) .
\end{aligned}
$$

CovScore and CosScore are the summed inverse co-variance distances and cosine similarities, transferred to the $[0,1]$ range applying the normalization $N^{0,1}$.

Our final feature value for the current feature $f_{i}$ is then

$$
v_{i}=\omega_{1} * \text { confidence }_{\theta}+\omega_{2} * \text { CovScore }+\omega_{3} * \text { CosScore }
$$

where $\omega_{1}, \omega_{2}, \omega_{3}$ are hyper-parameters. 
Once all features $F_{i} \notin F S$ have been scored and evaluated for their contribution toward class $\theta$ as part of set $F S$, we append the single feature that maximized $v_{i}$ to the set $F S$.

\section{EVALUATION}

Here we provide an empirical evaluation of our target-focused (TF) method compared with prevalent linear feature selection techniques:

- Mutual information (MI) is estimating statistical dependency for feature selection [23, 34] and is widely used as a non-parametric approach to evaluating data dependencies. The MI approach works by estimating the correlation level based on entropy from $k$-nearest neighbor distances.

- Max-relevance min-redundancy (mRMR) [33] and advanced mRMR (AmRMR) [18] are first-order incremental feature selection methods based on MI (mRMR) or Pearson's correlation (AmRMR) that eliminates redundancy in features while selecting relevant ones.

- The least absolute shrinkage and selection operator (Lasso) model [36] is an L1-based feature selection approach. Performing some regularization in addition to filtering out unwanted features, Lasso is an "automatic" approach to feature selection.

- Extremely randomized trees (Extra Trees) [10] is a tree-based model performing feature selection based on the importance values computed by the model.

- A budgeted cost-sensitive learning (CSL) approach [20] is a deep Q-networks-based approach for costsensitive feature acquisition.

- The DRL-based approach [17] is one that formalizes the problem as a Markov decision process (MDP) and solves it with linearly approximated Q-learning.

\subsection{Datasets}

We evaluated our model on the image classification task, as well as a breast cancer detection task, both chosen from the UCI Machine Learning Repository [7], in addition to various disease prediction tasks assembled using data from the National Health and Nutrition Examination Survey (NHANES) of the Centers for Disease Control and Prevention (CDC) [1].

For each of our sets, we select a target of special interest that we would like our model to focus on when deciding which features to acquire. Projecting this to the real world, the focus target will be a specific health issue in a dataset of symptoms and possible tests or images, pointing to more than one possible target class.

The data is as follows. From the UCI Machine Learning Repository, we use Statlog data, ${ }^{1}$ a dataset of evaluating image data and identifying a particular type of soil in satellite images. Also from UCI, we use the Breast Cancer Wisconsin dataset, ${ }^{2}$ providing features that are computed from a digitized image of a fine needle aspirate of a breast mass. From NHANES, we construct two datasets ourselves based on the approach described by Kachueee et al. [21]: one for evaluating diabetes and one for evaluating heart diseases. To construct our datasets, we join all possible NHANES tables that are correlated with our targets. For example, for the heart disease dataset, we join all tables that have features with correlation to any of five heart conditions. This causes the resulting sets to have a vast amount of possible features.

Dataset statistics, as well as the target chosen for each dataset, are listed in Table 1. For the NHANES datasets, targets are renamed from the original data for convenience. Blood glucose refers to the feature LBXGLU, the amount of glucose in the blood when fasting, used here to indicate whether or not an individual has diabetes. Congestive heart failure (CHF) refers to the feature MCQ160B, and it is one of five heart conditions for which we construct the dataset (MCQ160E, MCQ160F, MCQ160C, MCQ160B, MCQ180B).

\footnotetext{
${ }^{1}$ Available here: UCI Statlog (Landsat Satellite).

${ }^{2}$ Available here: UCI Breast Cancer Wisconsin. 
Table 1. Datasets Statistics

\begin{tabular}{cccccc}
\hline Dataset & Size & Features & Targets & Focus Target & Missingness \\
\hline UCI Breast Cancer & 569 & 32 & 2 & Malignant & $0 \%$ \\
UCI Statlog & 4,435 & 37 & 6 & Damp grey soil & $0 \%$ \\
NHANES Diabetes & 25,474 & 581 & 2 & Blood glucose & $25 \%$ \\
NHANES Heart & 49,346 & 555 & 5 & CHF & $25 \%$ \\
\hline
\end{tabular}

\subsection{Evaluation Methodology}

Assuming a constant budget for features, we run all feature selection approaches on the same training subset of the data and iteratively evaluate each feature we add. We select a single target to act as the focus of our method. We emphasize the model confidence for that specific target value that we wish to maximize overall targets. The target chosen for each dataset is listed in Table 1.

The compared models were constructed with the following parameters:

(i) MI between our training data and the training target was calculated using a different number of neighbors. Balancing the estimation variance and bias, we evaluated the number of neighbors $k \in[1,2,3,5,10]$. The $k=3$ instance, giving the best average result in all cases, was selected.

(ii) mRMR/AmRMR was evaluated on "MIQ," "MID," and "R value" feature evaluation methods.

(iii) Lasso with cross validation was used in this experiment. To find the best $\alpha$ value for the regularization process, we considered $\alpha \in[1,0.1,0.001,0.0005,0.0001]$. The best setup of Lasso for the average case was as follows: a maximum number of iterations was set to 1,000 , tolerance was set to 0.1 , and the number of cross-validation folds was set to 10 .

(iv) The Extra Trees classifier was used in our experiments. The number of estimators in this model was set to 1,000 , with no maximum depth defined. The minimum number of samples to split a node was set to 2, and the quality of split was measured by Gini impurity.

(v) CSL was evaluated using a Baysian L1 utility function. We ran the model for up to 40,000 epochs to allow the model time to learn. We stop the model when converged.

(vi) The DRL approach was evaluated using many models with different cost-accuracy trade-off hyperparameter values. We ran the model for up to 40,000 epochs to allow the model time to learn. We stop the model when converged.

(vii) Our TF feature selection was trained using $\omega_{1}=0.4, \omega_{2}=\omega_{3}=0.3$.

The machine used for evaluation had the following specifications: Intel 12-core i9-7920x (2.90-GHz) CPU, 128 GB of RAM, and four GeForce RTX 2080TI GPUs. Our models were written using Edward: A library for probabilistic modeling [38].

\subsection{Runtime Comparison}

Finding a globally optimal feature subset out of a collection of available features is an NP-hard problem. Instead of spending an exponential amount of time evaluating subsets, feature selection methods use heuristics to select an approximation of an optimal set. In our experiments, we compare with polynomial-time statistical methods (MI, mRMR/AmRMR, Lasso, Extra Trees) and deep learning approaches (CSL, DRL). Our proposed model trains a shallow machine learning model, using a single layer estimating the latent distribution, falling in the middle of these two groups in terms of complexity. In practice, taking the NHANES heart dataset as our baseline, we see Extra Trees and MI running fastest, returning within 30 minutes to 1 hour, followed by MI and mRMR/AmrMR taking about 1 to 2 hours. Our method and the deep learning methods take advantage of the GPUs on our machine since they heavily rely on linear computation. With a single GPU, our method takes between 2 and 3 hours to 
Table 2. Comparing F1 Scores for Feature Selection on Low Feature Count Sets

\begin{tabular}{c|ccccc|ccccc}
\hline \multicolumn{10}{c}{ F1 Scores } \\
\hline & \multicolumn{9}{c}{ Breast Cancer } & \multicolumn{5}{c}{ Statlog } \\
\hline f & MI & mRMR & Lasso & Extra Trees & TF & MI & mRMR & Lasso & Extra Trees & TF \\
\hline 5 & 0.93 & 0.94 & 0.80 & 0.91 & 0.95 & 0.70 & 0.27 & 0.35 & 0.08 & 0.86 \\
10 & 0.94 & 0.93 & 0.83 & 0.94 & 0.93 & 0.64 & 0.68 & 0.72 & 0.78 & 0.90 \\
15 & 0.93 & 0.94 & 0.88 & 0.95 & 0.94 & 0.80 & 0.70 & 0.85 & 0.78 & 0.90 \\
20 & 0.93 & 0.93 & 0.94 & 0.95 & 0.93 & 0.83 & 0.71 & 0.85 & 0.81 & 0.90 \\
25 & 0.93 & 0.93 & 0.94 & 0.95 & 0.94 & 0.83 & 0.77 & 0.87 & 0.81 & 0.91 \\
\hline
\end{tabular}

Note: $\mathrm{f}$ indicates the number of features acquired.

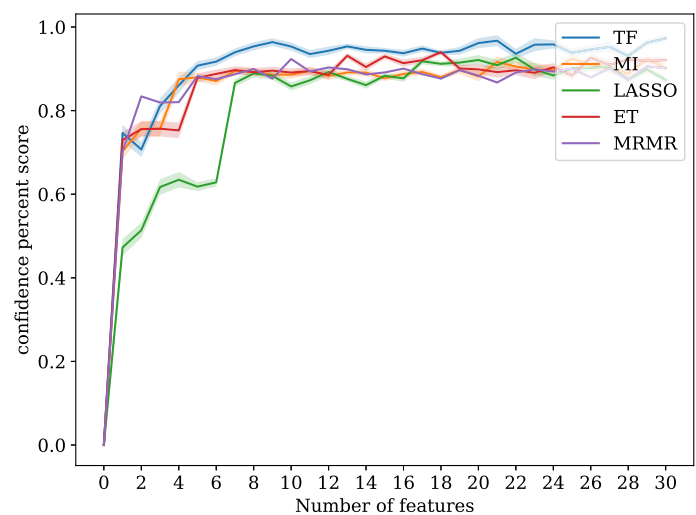

Fig. 1. Comparing model confidence in predicting malignant breast cancer. Line thickness indicates variance.

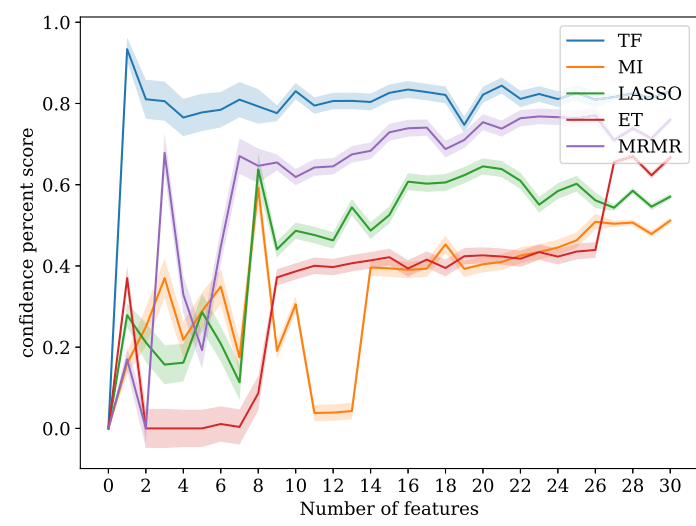

Fig. 2. Comparing model confidence in predicting one class out of the Statlog dataset. Line thickness indicates variance.

return. CSL takes approximately 3 days, and DRL takes 4 to 5 days to return results. Further time complexity discussion as well as a step by step algorithm can be found in Appendix A.

\section{RESULTS}

In this section, we report confidence, false-positive (FP) and false-negative (FN) scores, and F1 scores of our model at intervals as features are acquired. We separate our comparison to statistical methods for feature selection and deep learning approaches for sequential feature acquisition. When plotting model trends of the aforementioned metrics, we denote the variance scores of model confidence as the line margin on confidence plots, as can be seen in plots shown in the following.

\subsection{Comparing with Statistical Methods}

On datasets with low feature count and little missingness, our method was able to achieve better overall scores, faster than the comparable methods for a specific target value, as can be seen in Table 2 .

Figure 1 shows a confidence trend for acquiring 30 features on the Breast Cancer Wisconsin dataset using our method, comparing specific target confidence in the four compared models. In this case, our model can be seen to be on par with the confidence achieved by the MI and Extra trees methods. As can be seen in Table 2, our model presents slightly better F1 scores, indicating a faster FP/FN reduction. The breast cancer dataset proved to be a relatively simple prediction problem, and it can be seen that all methods performed relatively well, achieving good model confidence and F1 scores. 


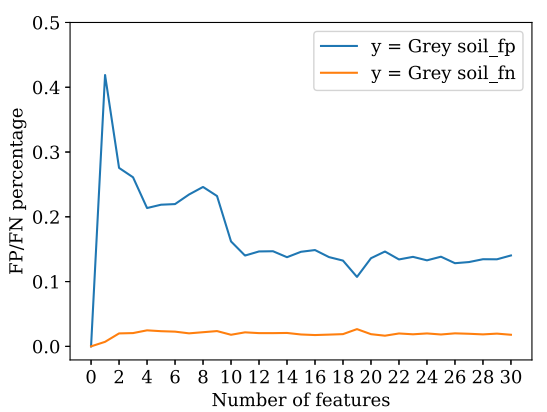

(a)

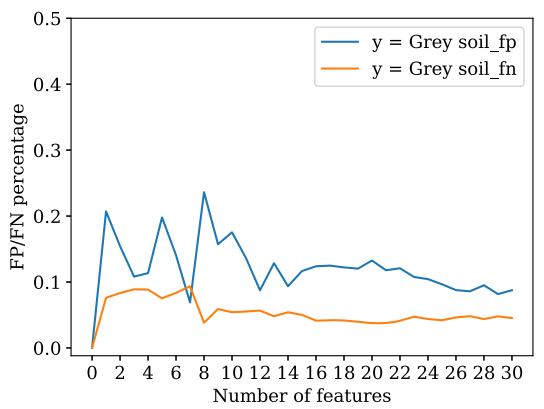

(c)

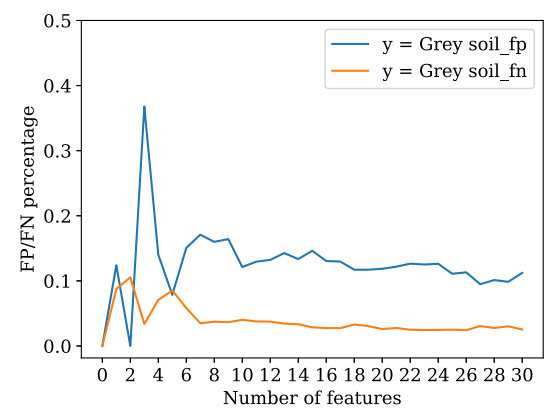

(b)

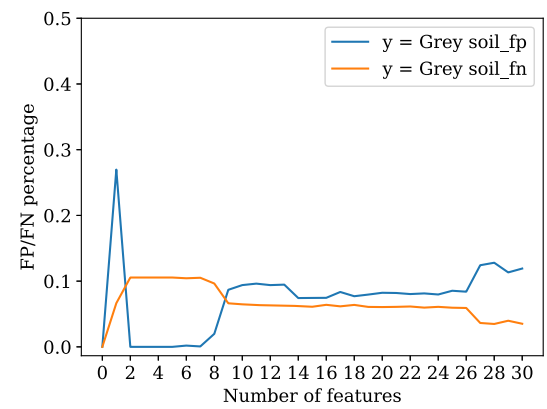

(d)

Fig. 3. Analysis of the UCI Statlog dataset comparing FP/FN rates as features are acquired. (a) Evaluation using our approach. (b) Evaluation using the mRMR "MIQ" method. (c) Evaluation using the Lasso method. (d) Evaluation using Extra Trees.

Table 3. Comparing F1 Scores for Feature Selection on High Feature Count Sets

\begin{tabular}{c|ccccc|ccccc}
\hline \multicolumn{10}{c}{ F1 Scores } \\
\hline & \multicolumn{9}{c}{ NHANES Diabetes } & \multicolumn{5}{c}{ NHANES Heart } \\
\hline f & MI & mRMR & Lasso & Extra Trees & TF & MI & mRMR & Lasso & Extra Trees & TF \\
\hline 5 & 0.76 & 0.79 & 0.61 & 0.79 & 0.92 & 0.66 & 0.55 & 0.28 & 0.39 & 0.72 \\
10 & 0.78 & 0.77 & 0.73 & 0.79 & 0.92 & 0.31 & 0.48 & 0.59 & 0.57 & 0.78 \\
15 & 0.80 & 0.79 & 0.77 & 0.77 & 0.92 & 0.78 & 0.69 & 0.56 & 0.32 & 0.87 \\
20 & 0.78 & 0.80 & 0.76 & 0.74 & 0.92 & 0.68 & 0.63 & 0.61 & 0.68 & 0.87 \\
25 & 0.76 & 0.80 & 0.64 & 0.77 & 0.92 & 0.85 & 0.76 & 0.66 & 0.47 & 0.86 \\
\hline
\end{tabular}

Note: $\mathrm{f}$ indicates the number of features acquired.

Figure 2 shows a confidence trend for acquiring 30 features on the Statlog dataset using our and compared methods. Here we can see that our TF method achieves a more confident model faster, as well as a better overall F1 score. In this case, the target of focus chosen appeared to be the hardest target to model out of the available targets, since all compared models struggled to find features that best model the data, in addition to it being one of the minority classes. Despite that, our model has gained the most confidence while using a low number of features. Figure 3 shows the FP/FN evaluation over the Statlog dataset. We see that our method shows a consistent non-volatile decline in FP rates while maintaining a low FN rate throughout.

In Table 3, we can see the F1 scores of the different feature selection methods compared on high feature count datasets, with missing values and lots of features. Here our model heuristic is evaluated on publicly available real-world healthcare data. Our method, being specific target aware, is able to pick out a good subset of the 


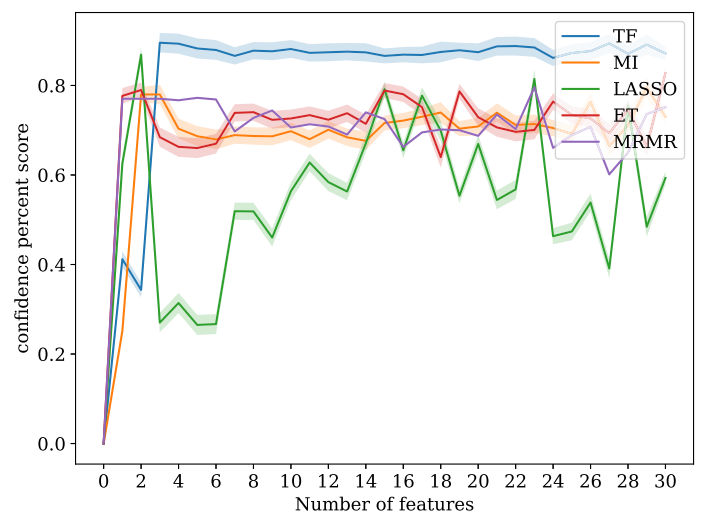

Fig. 4. Comparing model confidence in predicting diabetes. Line thickness indicates variance.

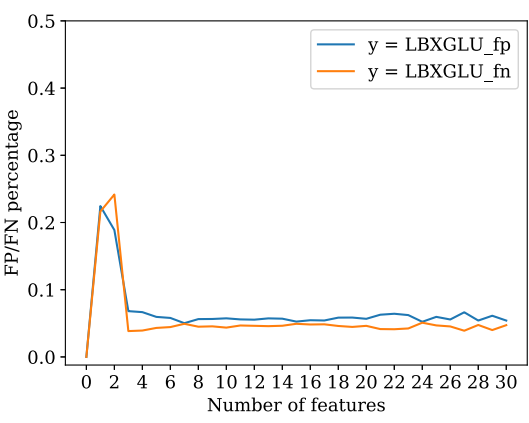

(a)

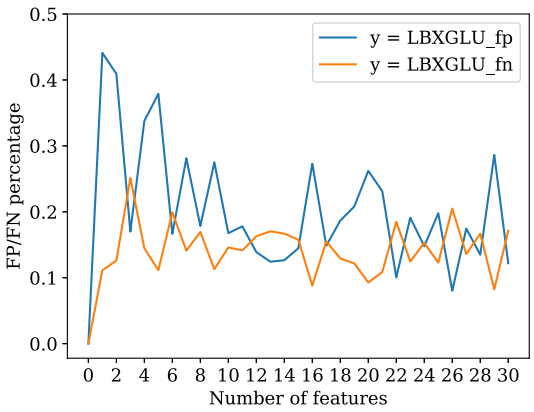

(c)

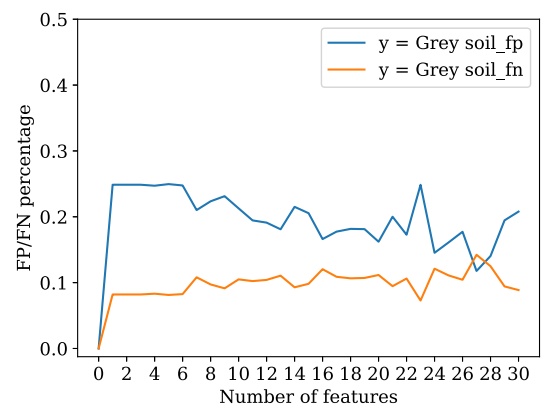

(b)

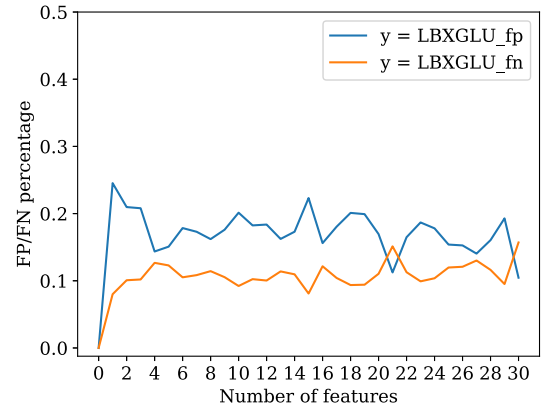

(d)

Fig. 5. Analysis of the NHANES Diabetes constructed dataset comparing FP/FN rates as features are acquired. (a) Evaluation using our approach. (b) Evaluation using the mRMR "MIQ" method. (c) Evaluation using the Lasso method. (d) Evaluation using Extra Trees.

features consistently, consequently using fewer features that in turn contribute most to maximizing the selected target class in focus.

As can be seen in the diabetes confidence evaluation in Figure 4, and in the FP/FN evaluation in Figure 5, our method outperformed the compared methods in minimizing FP and FN scores quickly, in addition to achieving a consistent amount of confidence in the target of interest relatively fast. It can be seen in Figure 4 that all 


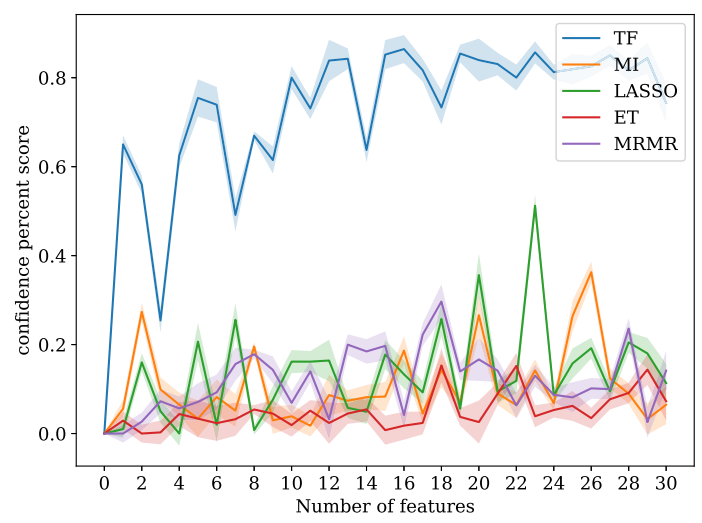

Fig. 6. Comparing model confidence in predicting CHF. Line thickness indicates variance.

Table 4. Comparing F1 Scores for Feature Selection on Low Feature Count Sets

\begin{tabular}{c|ccc|ccc}
\hline \multicolumn{7}{c}{ F1 Scores } \\
\hline & \multicolumn{3}{|c}{ Breast Cancer } & \multicolumn{3}{c}{ Statlog } \\
\hline f & CSL & DRL & TF & CSL & DRL & TF \\
\hline 5 & 0.91 & 0.76 & 0.95 & 0.00 & 0.23 & 0.86 \\
10 & 0.93 & 0.88 & 0.93 & 0.22 & 0.51 & 0.90 \\
15 & 0.92 & 0.86 & 0.94 & 0.49 & 0.35 & 0.90 \\
20 & 0.92 & 0.92 & 0.93 & 0.62 & 0.45 & 0.90 \\
25 & 0.95 & 0.86 & 0.94 & 0.70 & 0.10 & 0.91 \\
\hline
\end{tabular}

Note: $\mathrm{f}$ indicates the number of features acquired.

comparable methods achieve a high amount of confidence quicker than our TF method. However, comparing Figure 5(a) through (d), we can see that our model minimizes FP and FN scores quicker and therefore receives higher F1 scores.

Confidence evaluation for the heart disease dataset can be seen in Figure 6. Our model is gaining confidence using fewer features as before and keeps a relatively increasing trend of confidence. Other models failed to increase their confidence significantly, as this was the hardest task, with multiple targets and high dimensionality.

\subsection{Comparing with Deep Learning Methods}

As can be seen in Table 4, we achieve faster convergence on all datasets with little missingness and low feature count.

Similarly to the statistical methods comparison, our model can be seen gaining confidence faster than compared methods. Figure 7 shows a confidence trend for acquiring 30 features on the Breast Cancer Wisconsin dataset using our TF method, comparing specific target confidence in the three compared models. Our model can be seen gaining confidence slightly faster than the CSL method. As can be seen in Figure 8, the F1 score curve climb suggests faster learning of our target of interest using fewer features, in both our method and CSL. As our method gains confidence faster, it has a slight advantage on CSL in terms of observed performance on predicting our target.

Figure 9 shows a confidence trend for acquiring 30 features on the Statlog dataset using compared methods. For the task with high class count and low feature count, we are able to show much higher confidence convergence. Since the target chosen was the least frequent one, it appears that both deep learning approaches struggle to learn 


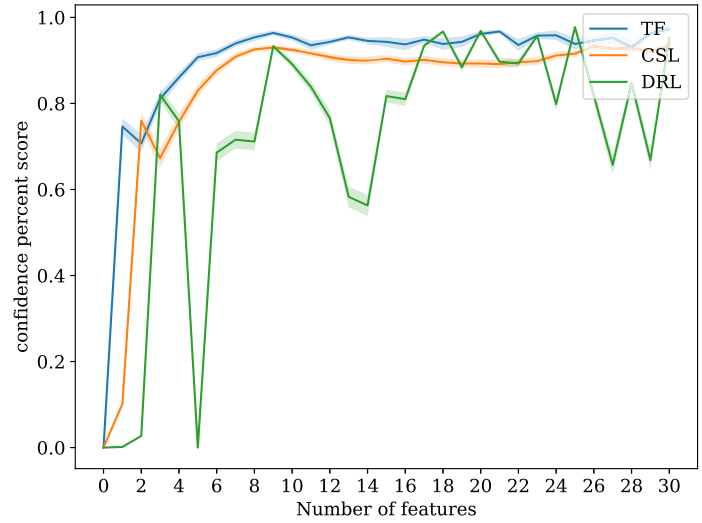

Fig. 7. Comparing model confidence in predicting malignant breast cancer. Line thickness indicates variance.

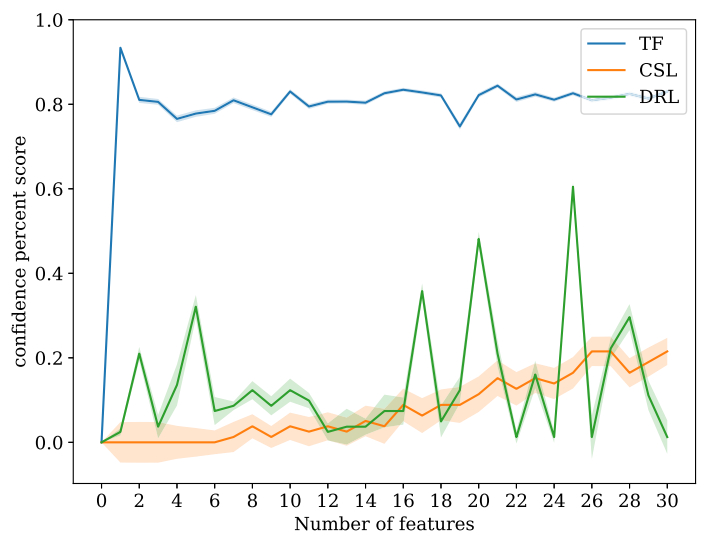

Fig. 9. Comparing model confidence in predicting one class out of the Statlog dataset. Line thickness indicates variance.

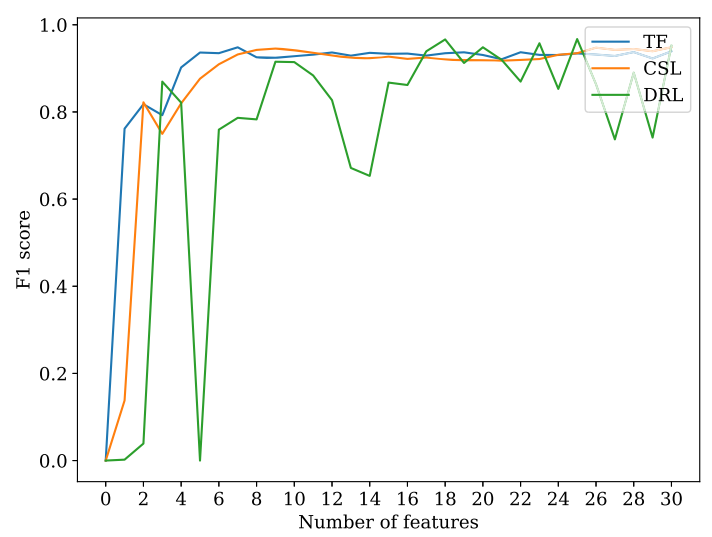

Fig. 8. Comparing the model F1 curve in predicting malignant breast cancer. Line thickness indicates variance.

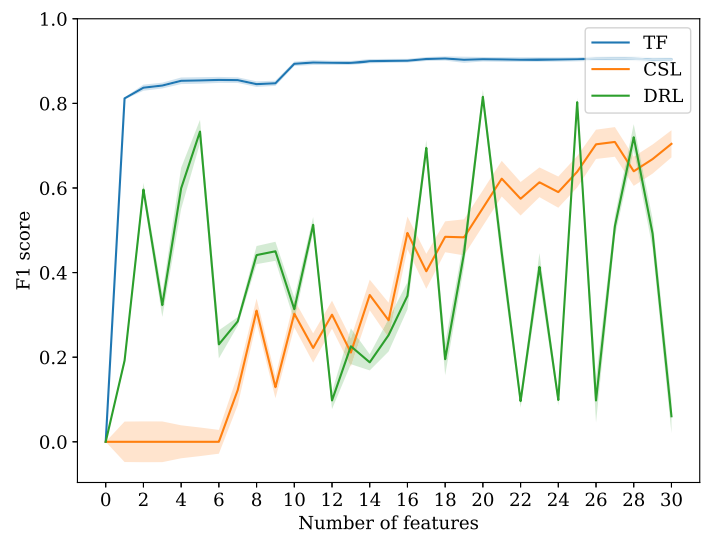

Fig. 10. Comparing the model $\mathrm{F} 1$ curve in predicting one class out of the Statlog dataset. Line thickness indicates variance.

the target given the amount of data available. Despite data shortage, our model has gained the most confidence while using a low number of features. Figure 10 shows the F1 score curve evaluation over the Statlog dataset. We see that our method shows a steady non-volatile gain, consistent with learning the target of interest with low FP and FN rates.

In Table 5, we can see the F1 scores of the deep learning feature selection methods along with our approach compared on high feature count datasets, with missing values and lots of features. Here our model heuristic is evaluated on publicly available real-world healthcare data. Our method is able to consistently outperform the deep learning methods in terms of F1 scores.

In Figure 11, we see a steady increase in confidence in our model in what is essentially the hardest type of task with multiple classes of heart conditions and a vast amount of features. Our TF approach is able to gain confidence efficiently toward the target of interest. Looking at the F1 curve in Figure 12 suggests that as both CSL and DRL continue to acquire features, they are not improving the overall performance on our target. Meanwhile, our 
Table 5. Comparing F1 Scores for Feature Selection on Low Feature Count Sets

\begin{tabular}{c|ccc|ccc}
\hline \multicolumn{7}{c}{ F1 Scores } \\
\hline & \multicolumn{2}{|c}{ NHANES Diabetes } & \multicolumn{3}{c}{ NHANES Heart } \\
\hline f & CSL & DRL & TF & CSL & DRL & TF \\
\hline 5 & 0.92 & 0.69 & 0.92 & 0.58 & 0.64 & 0.72 \\
10 & 0.90 & 0.89 & 0.92 & 0.48 & 0.33 & 0.78 \\
15 & 0.90 & 0.87 & 0.92 & 0.61 & 0.46 & 0.87 \\
20 & 0.89 & 0.89 & 0.92 & 0.51 & 0.63 & 0.87 \\
25 & 0.89 & 0.88 & 0.92 & 0.60 & 0.46 & 0.86 \\
\hline
\end{tabular}

Note: $\mathrm{f}$ indicates the number of features acquired.

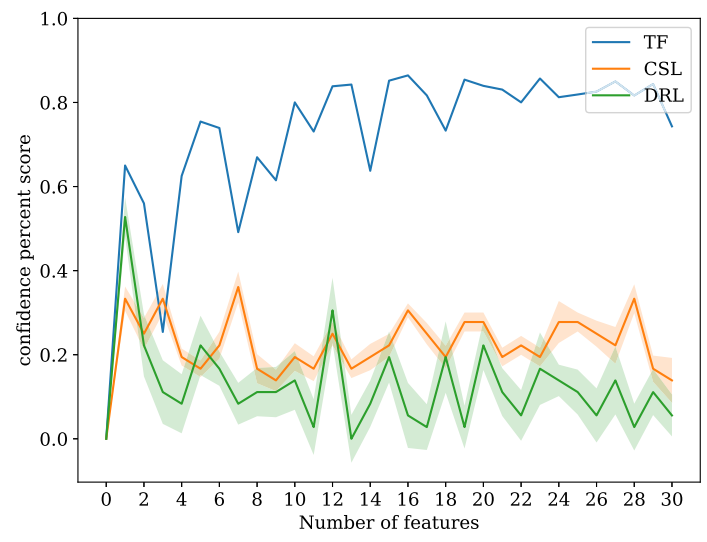

Fig. 11. Comparing model confidence in predicting CHF. Line thickness indicates variance.

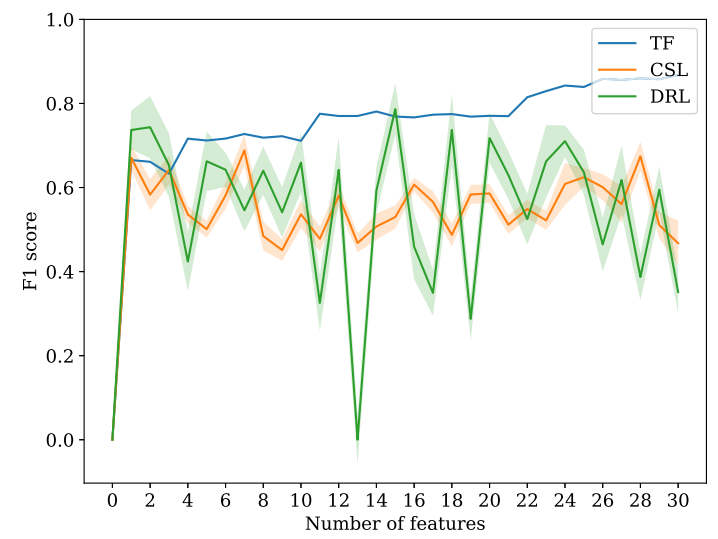

Fig. 12. Comparing the model F1 curve in predicting CHF. Line thickness indicates variance.

model is able to continuously acquire features that improve performance on the target of interest and receives higher F1 scores throughout.

Diabetes task confidence can be seen in Figure 13 and its corresponding F1 curve in Figure 14. Although DRL feature selection starts off with the most confidence, it can be seen in the F1 plot that the overall performance did not correspond with confidence scores, suggesting high FP rates for our target of interest. Conversely, both our approach and the CSL model did well in gaining confidence toward our target of focus, with our model yielding slightly higher F1 scores.

\subsection{Final Note}

Since the other feature selection models are unaware of the single target uncertainty in the model, the results obtained by the compared models could be largely dependent on the distribution of targets. In effect, the selected target of focus might get better results if it is also the majority target. All models compared were able to find features to construct an efficient frugal model on at least one of the sets, but our method has shown higher consistency across all sets. Although real-world health data is normally sparse and feature rich, we can see that even on smaller datasets with fewer features, our method provides a good heuristic as to the value of features when acquired toward a single target. 


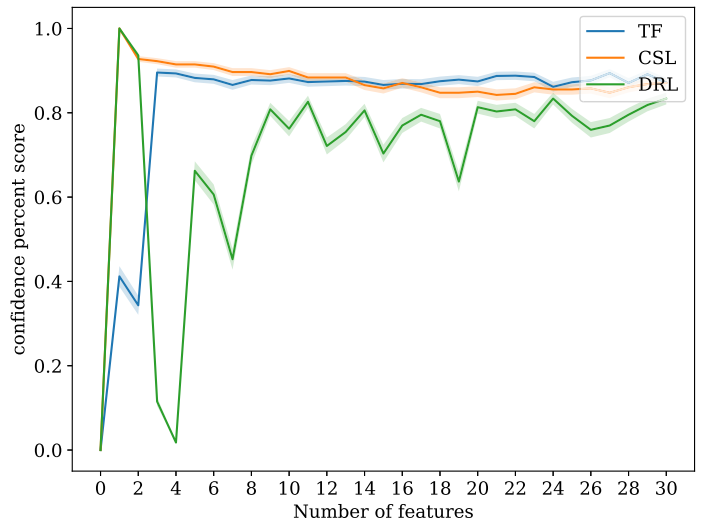

Fig. 13. Comparing model confidence in predicting diabetes. Line thickness indicates variance.

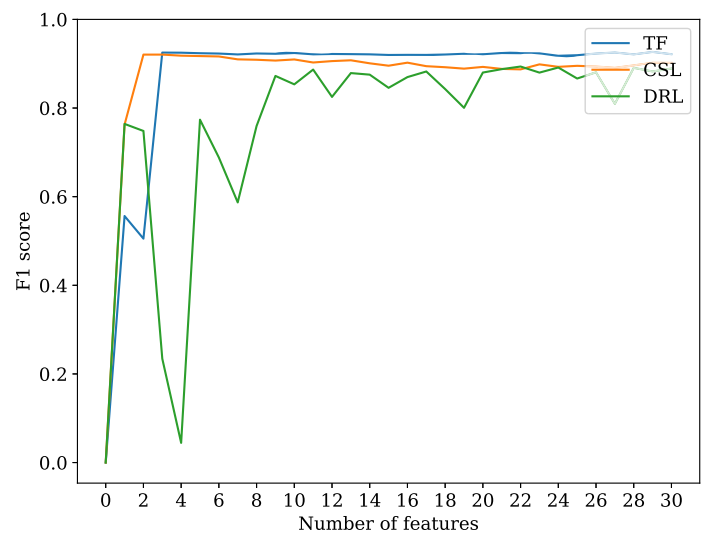

Fig. 14. Comparing the model F1 curve in predicting diabetes. Line thickness indicates variance.

\section{CONCLUSION}

In this article, we have investigated the approach of acquiring features based on a specific target of interest out of two or more targets. We see a frugal approach as an important addition to the process of feature selection, especially as data availability grows dramatically and utilization of data remains somewhat inefficient, particularly in the domain of healthcare. We have discussed the application of our TF approach to both well-known sources of machine learning datasets, as well as real-world public healthcare data converted into datasets. On these, we have demonstrated the value of having a target-aware method to feature selection as compared to feature selection methods that are target agnostic. We have introduced a Bayesian confidence-based scoring mechanism that we proceeded to show is robust in both scalability and consistency on different types of datasets. Practically, we were able to minimize uncertainty in a specific target of interest with a minimal budget while minimizing the general uncertainty, FP, and FN rates.

\section{APPENDICES}

\section{A TIME COMPLEXITY ANALYSIS}

Let $F S$ be the set collecting all selected features, $N$ the number of available features, and $D$ the number of data points in our traning set, and assuming some constant budget $\beta$ for features. For a single feature $f_{i} \notin F S$, we train a new model estimating the linear function $p\left(\mathbf{z}_{n} \mid \mathbf{X}_{n}^{\top} \mathbf{W}+\mathbf{b}, \sigma_{z}^{2}\right)$ (Equation (5) in the article). The model is trained using a constant number of iterations and confidence is computed using a constant number of samples from the estimated distribution. CovScore and CosScore are both computed on the features already in FS in time $2 * \beta^{2}$. Since $\beta$ is constant, so is the time to compute CovScore and CosScore. The final $v_{i}$ value is the product of another constant time multiplication.

Once all features are scored, we append a single feature to the set $F S$, and the process starts again for $N-1$ features. Therefore, for $N$ features, the process will run $N+N-1+N-2 \ldots+1=N^{2}$ times. Training a model using $D$ data points each time will result in $D N^{2}$ time complexity. We give a step-by-step description of our evaluation process in Algorithm 1. 


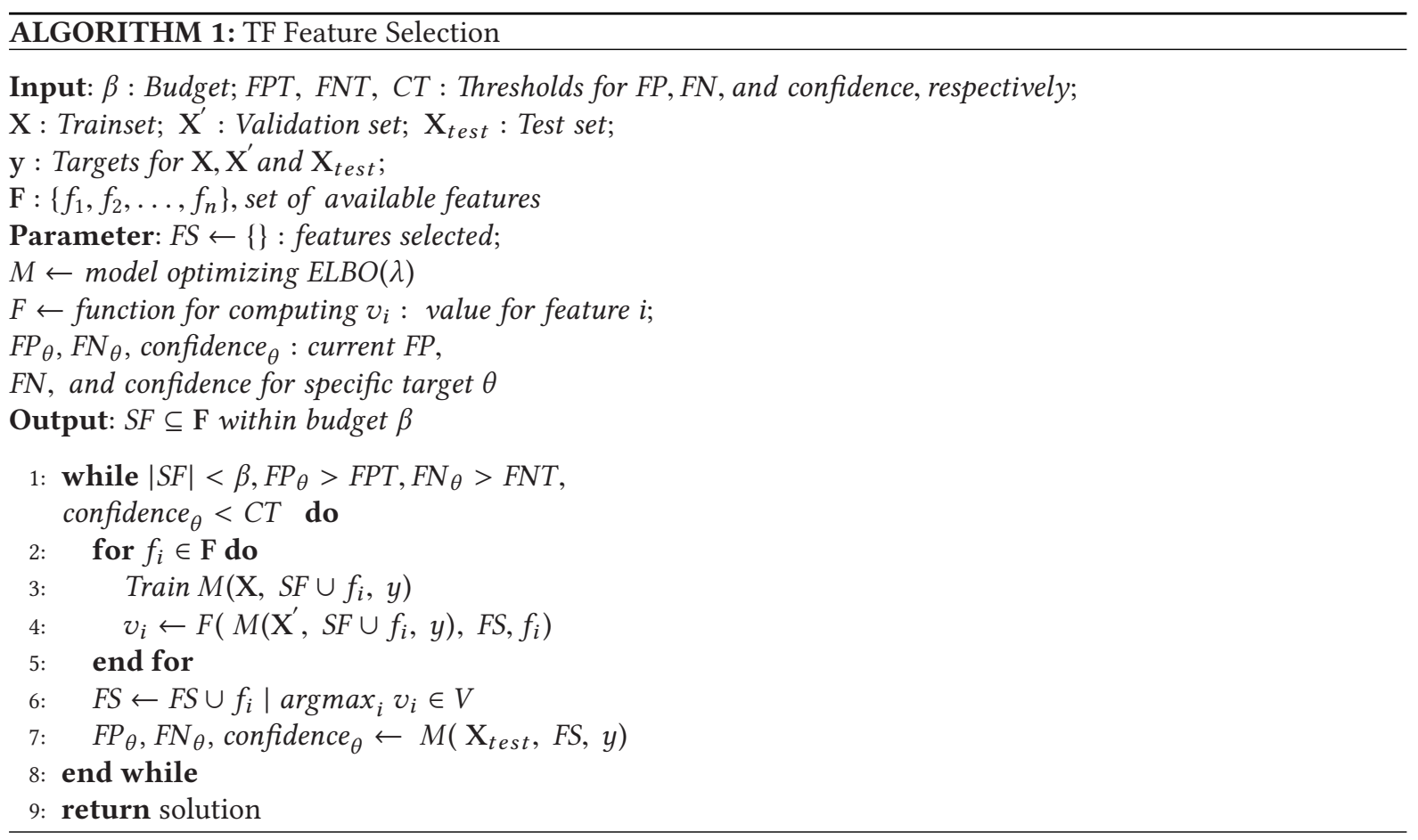

\section{B IMPACT ON HEALTHCARE DISCUSSION}

Development of learning models that will maximize confidence and acquire features while considering their costs could be the catalyst for the wider adoption of these models by health professionals. For certain diagnostics, the ability to accurately measure the FP/FN rates of a model greatly affects its usability in a healthcare setting. The ability to assist a healthcare provider's diagnostic abilities and decision-making process using a learning model is dependent on the provider's ability to trust such a model. Trust could be established if a model accurately advertises its expected statistical guarantees given the data that was fed into it. For example, as a physician is diagnosing a patient, feeding low-cost data (e.g., a standard medical questionnaire) into a machine learning model that is trained to diagnose a certain disease will keep track of a vast amount of possible tests that could be performed next on a patient. Additionally, the target-specific model will provide the expected gain toward a diagnosis or ruling out a hypothesis while considering the cost of all such tests.

Using a confidence-based feature selection method then allows for an informed decision-making process using actionable data from a machine learning model that can utilize much more data than is normally taken into account in a diagnosis process performed by a human. Training a target-specific model on as much expert knowledge as possible will allow this information to propagate and be used quickly in a machine learning context, where esoteric cases can be efficiently considered by selecting the most important features to acquire next.

\section{REFERENCES}

[1] Centers for Disease Control and Prevention. 2018. National Health and Nutrition Examination Survey. Retrieved March 30, 2020 from https://www.cdc.gov/nchs/nhanes.

[2] H. Bentz, M. Hagstroem, and G. Palm. 1997. Selection of relevant features and examples in machine learning. Neural Networks 2, 4 (1997), 289-293. 
[3] Christopher M. Bishop. 2006. Pattern Recognition and Machine Learning. Information Science and Statistics. Springer-Verlag, Berlin, Germany.

[4] Arthur Choi, Yexiang Xue, and Adnan Darwiche. 2012. Same-decision probability: A confidence measure for threshold-based decisions. International fournal of Approximate Reasoning 53 (2012), 1415-1428.

[5] YooJung Choi, Adnan Darwiche, and Guy Van den Broeck. 2017. Optimal feature selection for decision robustness in Bayesian networks. In Proceedings of the 26th International foint Conference on Artificial Intelligence (IFCAI'17).

[6] J. S. Denker and Y. LeCun. 1991. Transforming neural-net output levels to probability distributions. In Advances in Neural Information Processing Systems (NIPS'90), R. Lippmann, J. Moody, and D. Touretzky (Eds.), Vol. 3. Morgan Kaufman, Denver, CO.

[7] Dua Dheeru and Efi Karra Taniskidou. 2017. UCI Machine Learning Repository. Retrieved March 30, 2020 from http://archive.ics.uci. edu/ml.

[8] Alberto Freitas, Altamiro Costa-Pereira, and Pavel Brazdil. 2007. Cost-sensitive decision trees applied to medical data. In Proceedings of the International Conference on Data Warehousing and Knowledge Discovery. 303-312.

[9] Tianshi Gao and Daphne Koller. 2011. Active classification based on value of classifier. In Advances in Neural Information Processing Systems 24, J. Shawe-Taylor, R. S. Zemel, P. L. Bartlett, F. Pereira, and K. Q. Weinberger (Eds.). Curran Associates, Red Hook, NY, 1062-1070. http://papers.nips.cc/paper/4340-active-classification-based-on-value-of-classifier.pdf.

[10] Pierre Geurts, Damien Ernst, and Louis Wehenkel. 2006. Extremely randomized trees. Machine Learning 63, 1 (2006), 3-42.

[11] Zoubin Ghahramani. 2015. Probabilistic machine learning and artificial intelligence. Nature 521 (May 2015), 452-459. DOI : http://dx. doi.org/10.1038/nature14541

[12] Russell Greiner, Adam J. Grove, and Dan Roth. 2002. Learning cost-sensitive active classifiers. Artificial Intelligence 139, 2 (Aug. 2002), 137-174. DOI: https://doi.org/10.1016/S0004-3702(02)00209-6

[13] Peter Groves, Basel Kayyali, David Knott, and Steve Van Kuiken. 2013. The 'big data' revolution in healthcare. McKinsey Quarterly 2, 3 (2013), $1-17$.

[14] Isabelle Guyon and André Elisseeff. 2003. An introduction to variable and feature selection. fournal of Machine Learning Research 3 (March 2003), 1157-1182.

[15] Stefan Herzog and Dirk Ostwald. 2013. Sometimes Bayesian statistics are better. Nature 494, 7435 (2013), 35.

[16] Shamsul Huda, John Yearwood, Herbert F. Jelinek, Mohammad Mehedi Hassan, Giancarlo Fortino, and Michael Buckland. 2016. A hybrid feature selection with ensemble classification for imbalanced healthcare data: A case study for brain tumor diagnosis. IEEE Access 4 (2016), 9145-9154.

[17] Jaromír Janisch, Tomáš Pevnỳ, and Viliam Lisỳ. 2019. Classification with costly features using deep reinforcement learning. In Proceedings of the AAAI Conference on Artificial Intelligence, Vol. 33. 3959-3966.

[18] Insik Jo, Sangbum Lee, and Sejong Oh. 2019. Improved measures of redundancy and relevance for mRMR feature selection. Computers 8, 2 (2019), 42.

[19] Michael I. Jordan, Zoubin Ghahramani, Tommi S. Jaakkola, and Lawrence K. Saul. 1999. An introduction to variational methods for graphical models. Machine Learning 37, 2 (Nov. 1999), 183-233. DOI: https://doi.org/10.1023/A:1007665907178

[20] Mohammad Kachuee, Orpaz Goldstein, Kimmo Karkkainen, and Majid Sarrafzadeh. 2019. Opportunistic learning: Budgeted costsensitive learning from data streams. In Proceedings of the International Conference on Learning Representations. https://openreview. net/forum?id=S1eOHo09KX.

[21] Mohammad Kachuee, Kimmo Karkkainen, Orpaz Goldstein, Davina Zamanzadeh, and Majid Sarrafzadeh. 2019. Nutrition and health data for cost-sensitive learning. arXiv:1902.07102.

[22] Diederik P. Kingma and Max Welling. 2013. Auto-encoding variational Bayes. arxiv:1312.6114.

[23] Alexander Kraskov, Harald Stögbauer, and Peter Grassberger. 2004. Estimating mutual information. Physical Review E 69, 6 (2004), 066138.

[24] Martin Krzywinski and Naomi Altman. 2013. Points of significance: Importance of being uncertain. Nature Methods 10, 9 (Sept. 2013), 809-810.

[25] B. Lakshminarayanan, A. Pritzel, and C. Blundell. 2016. Simple and scalable predictive uncertainty estimation using deep ensembles. arxiv:stat.ML/1612.01474.

[26] Jundong Li, Kewei Cheng, Suhang Wang, Fred Morstatter, Robert P. Trevino, Jiliang Tang, and Huan Liu. 2018. Feature selection: A data perspective. ACM Computing Surveys 50, 6 (2018), 94.

[27] Peng Liu, Lei Lei, Junjie Yin, Wei Zhang, Wu Naijun, and Elia El-Darzi. 2006. Healthcare data mining: Prediction inpatient length of stay. In Proceedings of the 2006 3rd International IEEE Conference on Intelligent Systems. IEEE, Los Alamitos, CA, 832-837.

[28] Dijun Luo, Fei Wang, Jimeng Sun, Marianthi Markatou, Jianying Hu, and Shahram Ebadollahi. 2012. SOR: Scalable orthogonal regression for non-redundant feature selection and its healthcare applications. In Proceedings of the 2012 SIAM International Conference on Data Mining. 576-587.

[29] Dunja Mladenic and Marko Grobelnik. 1999. Feature selection for unbalanced class distribution and naive bayes. In Proceedings of the 16th International Conference on Machine Learning (ICML'99), Vol. 99. 258-267.

[30] Kevin P. Murphy. 2012. Machine Learning: A Probabilistic Perspective. MIT Press, Cambridge, MA. 
[31] Sriraam Natarajan, Srijita Das, Nandini Ramanan, Gautam Kunapuli, and Predrag Radivojac. 2018. On whom should I perform this lab test next? An active feature elicitation approach. In Proceedings of the 27th International foint Conference on Artificial Intelligence (IfCAI'18). 3498-3505. DOI : https://doi.org/10.24963/ijcai.2018/486

[32] Radford M. Neal. 1996. Bayesian Learning for Neural Networks. Springer-Verlag, Berlin, Germany.

[33] Hanchuan Peng, Fuhui Long, and Chris Ding. 2005. Feature selection based on mutual information: Criteria of max-dependency, maxrelevance, and min-redundancy. IEEE Transactions on Pattern Analysis \& Machine Intelligence 8 (2005), 1226-1238.

[34] Brian C. Ross. 2014. Mutual information between discrete and continuous data sets. PLoS One 9, 2 (2014), e87357.

[35] K. Srinivas, B. Kavihta Rani, and A. Govrdhan. 2010. Applications of data mining techniques in healthcare and prediction of heart attacks. International fournal on Computer Science and Engineering 2, 2 (2010), 250-255.

[36] Robert Tibshirani. 1996. Regression shrinkage and selection via the lasso. fournal of the Royal Statistical Society. Series B (Methodological) 58, 1 (1996), 267-288.

[37] Naftali Tishby, Esther Levin, and Sara A. Solla. 1989. Consistent inference of probabilities in layered networks: Predictions and generalization. In Proceedings of the International 1989 Joint Conference on Neural Networks. IEEE, Los Alamitos, CA, 403-409.

[38] Dustin Tran, Alp Kucukelbir, Adji B. Dieng, Maja Rudolph, Dawen Liang, and David M. Blei. 2016. Edward: A library for probabilistic modeling, inference, and criticism. arXiv:1610.09787.

[39] Jialei Wang, Peilin Zhao, Steven C. H. Hoi, and Rong Jin. 2014. Online feature selection and its applications. IEEE Transactions on Knowledge and Data Engineering 26, 3 (2014), 698-710.

[40] Yichuan Wang, LeeAnn Kung, and Terry Anthony Byrd. 2018. Big data analytics: Understanding its capabilities and potential benefits for healthcare organizations. Technological Forecasting and Social Change 126 (2018), 3-13.

[41] Stephen F. Weng, Jenna Reps, Joe Kai, Jonathan M. Garibaldi, and Nadeem Qureshi. 2017. Can machine-learning improve cardiovascular risk prediction using routine clinical data? PLoS One 12, 4 (2017), e0174944.

[42] Shipeng Yu, Balaji Krishnapuram, Romer Rosales, and R. Bharat Rao. 2009. Active sensing. In Proceedings of the 12th International Conference on Artificial Intelligence and Statistics. 639-646.

[43] Wei Zheng, Xiaofeng Zhu, Yonghua Zhu, and Shichao Zhang. 2018. Robust feature selection on incomplete data. In Proceedings of the 27th International foint Conference on Artificial Intelligence (IFCAI'18). 3191-3197. DOI : https://doi.org/10.24963/ijcai.2018/443

[44] Choong Ho Lee and Hyung-Jin Yoon. 2017. Medical big data: promise and challenges. Kidney Research and Clinical Practice 36, 1 (2017), 3.

Received February 2019; revised January 2020; accepted February 2020 